\title{
Moving insects on tympanic membrane
}

\author{
Wong Hui Tong* and Rajagopalan Raman \\ Department of Otorhinolaryngology, Faculty of Medicine, University of Malaya, 50603 Kuala Lumpur, Malaysia
}

\section{Introduction}

Foreign bodies of the external auditory canal (EAC) and tympanic membrane are frequently encountered by pediatricians, emergency care physicians, and otolaryngologists. The review of literature indicates an incidence of 1 in 219 to 1 in 17921, with a 73\% pediatric affliction rate [1].

Various foreign body can be found in the ear including cotton, beads, paper, toys, eracers, beans, popcorn kernels and insects [2]. Insects account for 14-18\% of aural foreign body cases [2,3].

We present an interesting case of tiny insects moving on the tympanic membrane causing symptoms of foreign body sensations.

\section{Case}

A 45-year-old woman presented with a 1-day history of foreign body sensation in the right ear. She can feel an insect moving in the ear. Initial otoscopic examination could not identify the problem as the insect is very small. We then proceed to endoscopic examination of the right ear and it shows normal external auditory canal and a small insect moving in the tympanic membrane (Figure 1). Olive oil was used to fill the EAC and to kill the insects. Irrigation was then done to remove the insect. The offending organism species could not be specified.

\section{Discussion}

The term "insect" was used to include cockroaches beetles and arachnids, such as spiders and ticks. Cockroaches were the most commonly specified insect foreign bodies (78\%). In nearly $38 \%$ of insect foreign body cases, the offending organism was not specified [3].

The insects will cause pain and a very distressing tinnitus. Therefore, accurate diagnosis and rapid initiation of management is important. Occasionally the foreign body is so small that can be missed on microscopic and ootoscopic examination. In such a situation, an oto-endoscope is recommended.

The best solution is one that can provide rapid inactivation (killing) of insects and yet do not cause over-activity of insects prior to the death. Various solutions have been recommended for killing of the insects. The best solution from Leffler et al is microscope immersion oil (mineral oil) [4], with similar effect from olive oil or baby oil. Ticks are exception and resistant to various solutions [3]. Using lidocaine can occasionally lead to serious vertigo which require admissions [5].

In conclusion, oto-endoscopic examinations can show the tiny insects and increase diagnostic accuracy. Mineral oil or olive oil is the best solution to rapidly kill the insect and removal can be done using forceps or saline flushing.

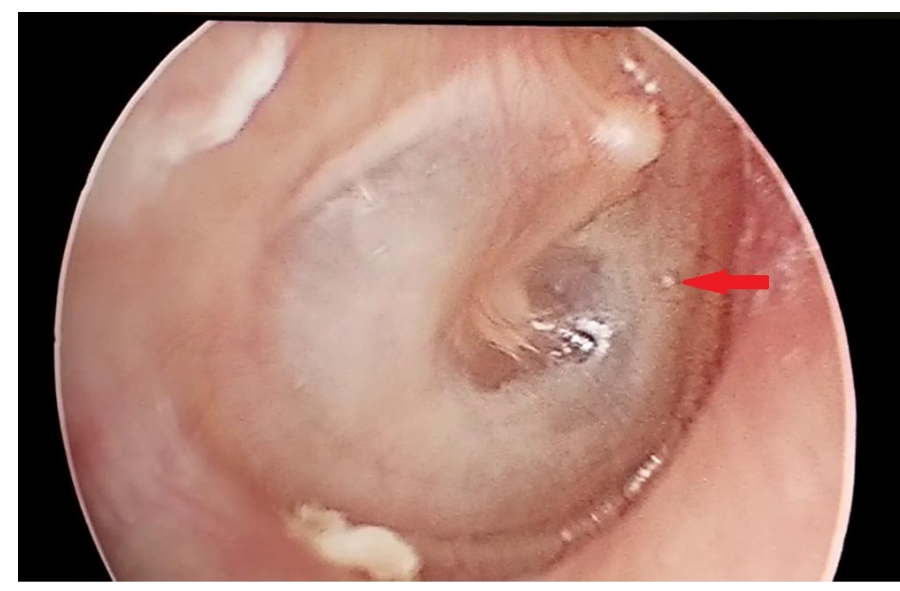

Figure 1. Endoscopic view of right ear showing the insect foreign body $(\Rightarrow)$.

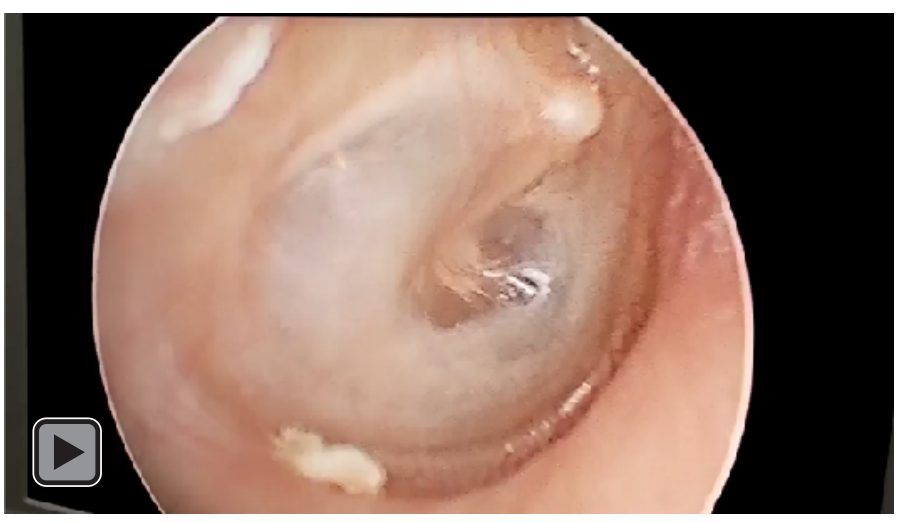

Video. Endoscopic view showing insect moving on the tympanic membrane.

Correspondence to: Wong Hui Tong, Department of Otorhinolaryngology, Faculty of Medicine, University of Malaya, 50603 Kuala Lumpur, Malaysia, Tel: 03-79492062, Fax: 03-79556963,E-mail: whtong83@gmail.com

Received: January 02, 2018; Accepted: January 22, 2018; Published: January 24, 2018 


\section{References}

1. Moffat DA (1998) Temporal bone trauma. In: Ludmen H, Wright T, editors. Diseases of the ear. 6th ed. London7 Arnold; p. 448.

2. Thompson SK, Wein RO, Dutcher PO (2003) External auditory canal foreign body removal: management practices and outcomes. Laryngoscope 113: 1912-1915. [Crossref]
3. Antonelli PJ, Ahmadi A, Prevatt A (2001) Insecticidal activity of common reagents for insect foreign bodies of the ear. Laryngoscope 111: 15-20. [Crossref]

4. Leffler S, Cheney P, Tandberg D (1993) Chemical immobilization and killing of intraaural roaches: an in vitro comparative study. Ann Emerg Med 22: 1795-1798. [Crossref]

5. Kizer KW (2000) Intra-aural insects, lidocaine, and vertigo. Wilderness Environ Med 11: 138-139. [Crossref]

Copyright: (C2018 Tong WH. This is an open-access article distributed under the terms of the Creative Commons Attribution License, which permits unrestricted use, distribution, and reproduction in any medium, provided the original author and source are credited. 